Motion increases recognition of spontaneous postures but not facial expressions

Van Der Zant, T. ${ }^{1} \&$ Nelson, N. L. ${ }^{2}$

The University of Queensland ${ }^{1}$

The University of Adelaide ${ }^{2}$

Word Count: 4848

Address for correspondence:

Tamara Van Der Zant, School of Psychology, The University of Queensland, St Lucia, QLD, 4067, Australia. Email: t.vanderzant@uq.edu.au

Conflict of Interests: The authors declare they have no conflict of interest. 


\begin{abstract}
Although emotion expressions are typically dynamic and include the whole person, much emotion recognition research uses static, posed facial expressions. In this study, we created a stimulus set of dynamic, naturalistic expressions drawn from professional tennis matches to determine whether movement would result in better recognition. We examined participants' judgments of static vs. dynamic expressions when viewing an isolated face, an isolated body, or a whole person. Dynamic expressions increased recognition of whether the player had won or lost the point. In addition, recognition improved when the whole person was presented as opposed to only the face or body. However, overall recognition of wins and losses was poor, with recognition for isolated faces being poorer than chance for winning players. Our findings highlight the importance of incorporating dynamic stimuli and support previous research showing that recognition of naturalistic expressions differs greatly from the commonly-used posed and isolated facial expressions of emotion. Using a wider range of naturalistic stimuli should be incorporated into future research to better understand how emotion recognition functions in daily life.
\end{abstract}

Key Words: Emotional Expressions; Naturalistic Emotional Expressions; Real-World Expressions 


\section{Motion increases recognition of spontaneous postures but not facial expressions}

The vast majority of research in emotion expression and perception is conducted using posed, static facial expressions (Aviezer, Ensenberg, \& Hassin, 2017). However, expressions of emotion in daily life are typically moving, conveyed with the face as well as the body, hands and voice, are observed in context, and spontaneously generated rather than deliberately posed. As the field of expression research rises to meet the challenge of how expression and perception of emotion occurs with more ecologically valid stimuli, research has begun to explore how features such as motion and context may alter perceptions of emotion compared to static images of faces devoid of context. Research that has examined naturalistic emotional expressions has found evidence that these expressions are highly variable and poorly recognized (Abramson et al., 2017; Barrett et al., 2019; Duran \& Fernández-Dols, 2018). In this study, we examine differences in how emotional facial expressions are perceived when presented dynamically vs. statically, and with vs. without the accompanying bodily expression.

\section{Naturalistic vs Posed Expressions}

Research examining naturalistic spontaneous expressions of emotion have found wide variation in expressive behaviour (Sauter \& Fischer, 2018). Naturalistic expressions are more poorly recognised than highly standardised posed expressions (Abramson et al., 2017; Crivelli et al, 2017; Dawel et al, 2019; Sauter \& Fischer, 2018), likely because they are often more subtle and ambiguous than posed expressions (Duran et al., 2017; Fernández-Dols \& Crivelli, 2013; Reisenzein et al., 2013). Specific features of genuinely elicited and posed expressions, when compared, differ in various ways, including showing distinct patterns of facial movements (Fernández-Dols et al., 1997; Namba et al., 2021; Schützwohl \& Reisenzein, 2012) and distinct temporal patterns of movement (Hess \& Kleck, 1990; Namba et al., 2021). When comparing spontaneous expressions and the emotions people report 
feeling in that moment, the expressions often do not align with the hypothesized expressions proposed in previous literature (Fernández-Dols \& Crivelli, 2013; Kraut \& Johnston, 1979; Reisenzein et al., 2006, 2013; Ruiz-Belda et al., 2003).

If posed expressions of emotion do not consistently resemble genuine and naturalistic expressions of emotion, then the study of emotion perceptions using such expressions fails to inform how typical expressions are perceived. In this study we developed a set of expressions from a naturalistic setting (professional tennis matches) aligned with clear experiences of great success or failure (win or loss of a major point, respectively) to assess patterns of emotion expression and perception. Winning expressions have been found to be rated as more positive than losing expressions in previous literature (Aviezer et al., 2012a). This set of expressions adds to the expanding evidence regarding how emotion expressions appear in various naturalistic contexts (Abramson et al., 2017; Aviezer et al., 2012a; Cowen \& Keltner, 2019; Douglas-Cowie et al., 2011; Wenzler et al., 2016).

\section{The Interaction of Face and Body Cues}

The face has been central to research of emotion expression and perception with contextual cues, including expressive behaviour of bodies, often excluded. However, this approach fails to account for the fact that faces are generally viewed in context and with the body (Aviezer, Ensenberg, \& Hassin., 2017; Dukes et al., 2017), and emotion expressed by the body is equally (or better) recognized than emotion expressed by the face (Abramson et al., 2017; Aviezer et al., 2012a; De Gelder, 2009). In addition, expressions are most accurately identified when both faces and bodies are available (Abramson et al., 2017; Martinez et al., 2016). Some research suggests faces and bodies may communicate different categories of emotion more clearly (Abramson et al., 2017; Martinez et al., 2016) or may communicate emotion more clearly in different contexts (Abramson et al., 2017; Aviezer et al., 2012a). 
The perception of faces is also influenced by the presence of bodies or other contextual features, even when participants are deliberately attempting to ignore non-facial information (Aviezer et al., 2011, 2012b; Lecker et al., 2017; Mondloch et al., 2013; Nelson \& Mondloch, 2017). Therefore, emotion perception from faces presented alone not only omits important expressive behaviour of the body typically used in emotion perception, but may inaccurately characterise typical perception of facial expressions as these are not perceived independent of bodies but are influenced by bodily expressions.

Like faces, expressions of the body differ when posed vs naturalistic expressive behaviour (Abramson et al., 2017). As such, the influence of bodily expressions on global perceptions of emotion may be different with naturalistic expressions than posed expressions (Abramson et al., 2017; Aviezer et al., 2012a). Examining how naturalistic whole person expressions are perceived allows better understanding of the generalisability of findings of research studying isolated facial or bodily expressions or posed whole person expressions.

\section{Expressions in Motion}

As with the absence of body expressions, the absence of motion in examining emotion expression perception eliminates a defining feature of expressive behaviour. Perception of emotion is enhanced by presenting expressions in motion rather than in static form (Ambadar et al., 2005; Atkinson et al., 2004; Krumhuber et al., 2013; Nelson \& Mondloch, 2017). Even motion alone, presented as point-light displays with the face and body features invisible, can convey emotion to perceivers (Atkinson et al., 2004). Motion may also increase perceptions of emotional intensity and arousal (Krumhuber et al., 2013; Barker, Bidstrup, Robinson \& Nelson, 2020). Recent research examining why dynamic stimuli enhance expression recognition has found that the benefit does not stem from the additional static information being provided via an increased frame rate, but rather due to the perception of movement 
across the frames presented (Bould \& Morris, 2008). Specifically, when the perception of movement is interrupted by placing visual masks between frames, recognition decreases.

Deliberately posed expressions also contain different patterns of motion than expressions that are spontaneously generated (Namba et al., 2017, 2021). Examining specific physical components of various emotion expressions has demonstrated differences in which expression components are present in spontaneous vs posed expressions, but also differences in the temporal patterns of movement (Namba et al., 2017, 2021). These differences may also explain why presenting expressions dynamically rather than statically improves participants ability to detect whether expressions were genuine or posed (Namba et al., 2018; Zloteanu et al., 2018).

\section{The Current Research}

This study examines how naturalistic, dynamic, whole person expressions are perceived. Specifically, we seek to build on the work of Aviezer et al. (2012a) which examined naturalistic whole person expressions of emotion. In this work they found that when viewing photographs of naturalistic, isolated faces, participants were unable to differentiate even the valence of the expressions (Aviezer et al., 2012a), a finding unusual in the expression recognition literature (Abramson et al., 2017; Sauter \& Fischer, 2018). However, when viewing the players' isolated bodies, or faces and bodies together, participants' judgments were more accurate (Aviezer et al., 2012a). This aligns with other literature suggesting that naturalistic bodily expressions may be more influential for recognition, as naturalistic facial expressions are more subtle and ambiguous than posed ones (Abramson et al., 2017; Aviezer et al., 2012a).

We build on Aviezer et al's (2012a) work by examining how motion influences the perception of naturalistic facial and bodily expressions. A recent review of available sets of 
dynamic emotion expressions listed no naturalistic sets which included the whole body, and the only set including body expression used posed expressions (Krumhuber et al., 2017). Thus, our work addresses a notable gap in the emotion literature, as well as to the ecological validity of the emotion recognition research.

To build our stimulus set, we followed a rigorous process of stimuli selection to ensure our results were generalisable. The research by Aviezer et al. (2012a) used images from of highly intense face and body expressions of professional tennis players following the win or loss of a point in an important match identified via a Google search. The authors acknowledge this may have led to selection of only the most interesting and intense reactions as selected by sports journalists, rather than the most typical expressions for the setting (Aviezer et al., 2012a). To address this concern, we selected expressions from the same naturalistic context - professional tennis players winning or losing a point - using video recordings of full matches. This allowed us to select all available expressions that met our criteria, not just those expressions selected by journalists or publications as being engaging or interesting. Thus, we were able to examine a more complete range of expressive behaviours typical in such intense emotional contexts.

For this study, we collected participants' perceptions of whether the player had won or lost the prior point in the game, a rating of perceived valence, and a rating of perceived arousal. We expected participant's ratings of valence and judgements of whether the player had previously won or lost the point to be closely related as we anticipated that judgements of player success would be informed by perceived valence. Arousal ratings were collected for the purpose of engaging in explorative analyses as little prior research was available to adequately inform hypotheses. 
We preregistered two specific hypotheses based on prior research. 1) We expected perceptions of Body-Only stimuli to be more accurate than Face-Only stimuli. Therefore, we expected (a) judgements regarding whether the player had won or lost the prior point to be more accurate for Body-Only expressions than Face-Only expressions and (b) winning and losing expressions to be judged as more positively and negatively valenced, respectively, when perceived from Body-Only expressions than from Face-Only expressions. 2) We also expected that dynamic stimuli would be better recognised than static stimuli. This would be evidenced by (a) greater accuracy for success judgements when stimuli are dynamic rather than static, and (b) the valence of winning expressions being perceived more positively valenced and losing expressions perceived as more negatively valenced when presented dynamically rather than statically.

The research also provides ample opportunity to examine exploratory questions which do not have adequate literature to merit hypothesis testing. We examined whether the effect of motion varies for different stimuli types (i.e., Face-Only, Body-Only, Face-Body), whether winning and losing expressions were similarly recognisable, and how arousal was perceived across stimuli types and with or without motion.

The preregistration document for this study can be found here: https://osf.io/qgfhk/?view only=b77e3c4d3b324265bfe5235f2538cfcc, and all stimuli and data are available on the Open Science Framework here:

https://osf.io/ay8x9/?view_only=3f2de1e7915640609831bb80a664a9c4 [anonymised links for blinded peer review]. Deviations from preregistration are detailed in the Supplemental Materials. This study was approved by the [removed for blind review] Ethical Review Board.

\section{Method}

\section{Participants}


Participants were one hundred and thirty-two undergraduate psychology students (age: 17 - 53 years, $\mathrm{M}=19.7$ years, $\mathrm{SD}=4.23 ; 96$ female, 35 male, 1 other) who participated in exchange for course credit. An a priori power analysis using $\mathrm{G}^{*}$ Power software (Faul et al., 2007) determined that to find a small effect $(f=.15, \alpha=.05$, power $=.95)$ in our primary analysis of a $2 \times 2 \times 3$ repeated measures ANOVA, a sample size of 120 participants was necessary, a number we exceeded.

\section{Stimuli Selection}

Stimuli were face and body expressions sourced from videos of significant tennis matches. To ensure matches were important, our initial search included all Grand Slam tournaments played between the 2017 and 2014 Australian Open tournaments. We next selected matches from within these tournaments that were important, and thus likely to evoke significant emotion, limiting our search to Finals, Semi-Finals, and Quarterfinals matches. We excluded matches in which complete broadcasts could not be found, or video quality was low (under 360p). See the Supplemental Materials for more stimuli details.

We focused on expressions stemming from deciding moments in the match and included all expressions that were: 1) visible for .5 seconds or more, 2) showed at least $50 \%$ of the player's face and their torso, 3) were visible within 2 seconds of the end of the point, and 4) the player's face was larger than $1.5 \mathrm{~cm}$ on a $53 \mathrm{~cm} \times 30 \mathrm{~cm}$ screen. We also ensured that from each match, a given player was shown in a winning point and a losing point.

Our stimulus set included 72 unique expressions - 36 winning points and 36 losing points - with expressions drawn from 14 different players (6 female, 8 male, ages 24-37) across 13 Grand Slam matches.

\section{Stimulus Creation and Study Design}

The background was removed from each Face-Body stimulus using Adobe AfterEffects. The player, their clothes, and their rackets were all retained in the final stimulus 
set. Next, the dynamic Face-Body stimuli were used to create static stimuli. A still frame was selected from each video clip, at the apex of the face and body expression.

To create the Face-Only and Body-Only stimuli, the faces and bodies of the FaceBody stimuli were isolated using Adobe After Effects for dynamic stimuli and PhotoShop for static stimuli. We included the racket being held by players, as removing it gave players the appearance of having a clenched fist (rather than gripped racket) and could have impacted participants' emotion judgments. This process resulted in a total of 432 stimuli, with 72 of each type of stimulus: Dynamic Face-Body; Dynamic Face-Only; Dynamic Body-Only; Static Face-Body; Static Face-Only; and Static Body-Only stimuli (Figure 1).
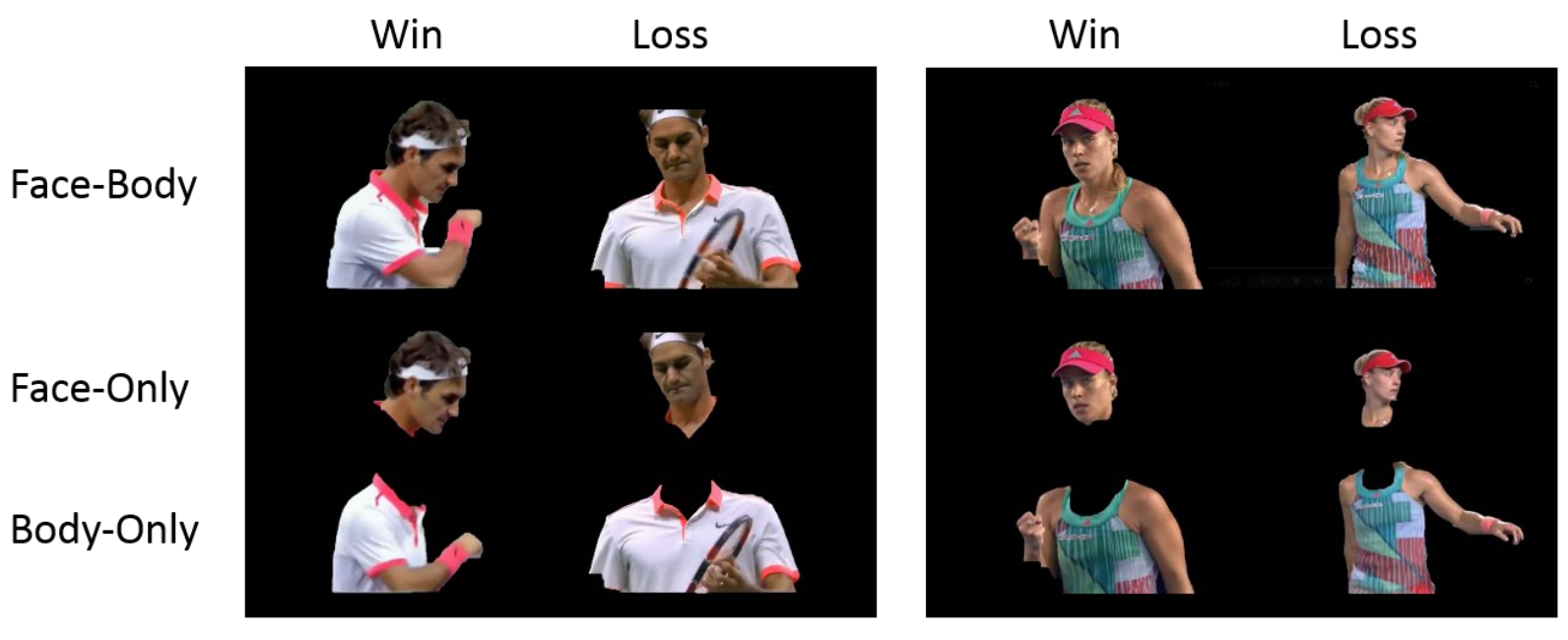

Figure 1.

Example of stimuli types.

To allow each participant to view all six forms of stimuli without viewing the same expression more than once (thus, potentially altering their emotion judgments), stimuli were divided into four sets (Sets A-D). Each set included 18 expressions and could be presented as dynamic or static, and as Face-Only, Body-Only or Face-Body. Each set included equal proportions of wins and losses and a similar proportion of male and female players' expressions. The sets were distributed across four surveys using a Latin Square design. Table 
1). Within each survey blocks were presented in a random order and stimuli within each block stimuli were presented in a random order.

Table 1.

Design of surveys.

\begin{tabular}{llllll}
\hline Motion & Cue Type & $\begin{array}{l}\text { Survey } \\
\text { One }\end{array}$ & $\begin{array}{l}\text { Survey } \\
\text { Two }\end{array}$ & $\begin{array}{l}\text { Survey } \\
\text { Three }\end{array}$ & $\begin{array}{l}\text { Survey } \\
\text { Four }\end{array}$ \\
\hline Dynamic & Face-Body & Set A & Set D & Set C & Set B \\
& Body-Only & Set B & Set A & Set D & Set C \\
& Set B & Set A & Set D & Set C \\
& Face-Body & Set C & Set B & Set A & Set D \\
Static & Face-Only & Set D & Set C & Set B & Set A \\
& Body-Only & Set D & Set C & Set B & Set A \\
\hline
\end{tabular}

\section{Procedure}

Participants were randomly assigned to complete one of the four surveys. All surveys were presented on a 53 × $30 \mathrm{~cm}$ computer screen with a screen resolution of 1024 x 768 pixels and a refresh rate of $30 \mathrm{fps}$. Participants viewed each stimulus, one at a time, with no time constraints. Dynamic videos could be replayed if participants wished and static images remained on screen; stimuli were available until participants made a response.

Participants viewed each stimulus twice, once while judging the player's success, and once while judging the valence and arousal of the expression. In judging the players' success, participants were simply asked, 'Did the player win or lose?' to which they were given the options 'The player won' or 'The player lost'. In judgements of valence and arousal participants were asked 'How is this person feeling?' before being instructed to choose a response from each scale. The scales were seven-point Likert type scales ranging from 'Very 
Negative' (-3) to 'Very Positive' (+3) for the valence scale and 'Very Sleepy' (-3) to 'Very Awake' (+3) for the arousal scale. The order of presentation for success, valence, and arousal judgements was counterbalanced.

\section{Results}

In this section we focus on participant's judgments of valence and accuracy as the measures relevant to our pre-registered hypotheses, and present exploratory analyses of valence, accuracy, and arousal. Additional analyses, including an analysis of sensitivity $(d ')$ and bias $(c)$ judgements can be found in the Supplemental Materials. Average valence and arousal scores for each stimulus type are plotted in Figure 2.

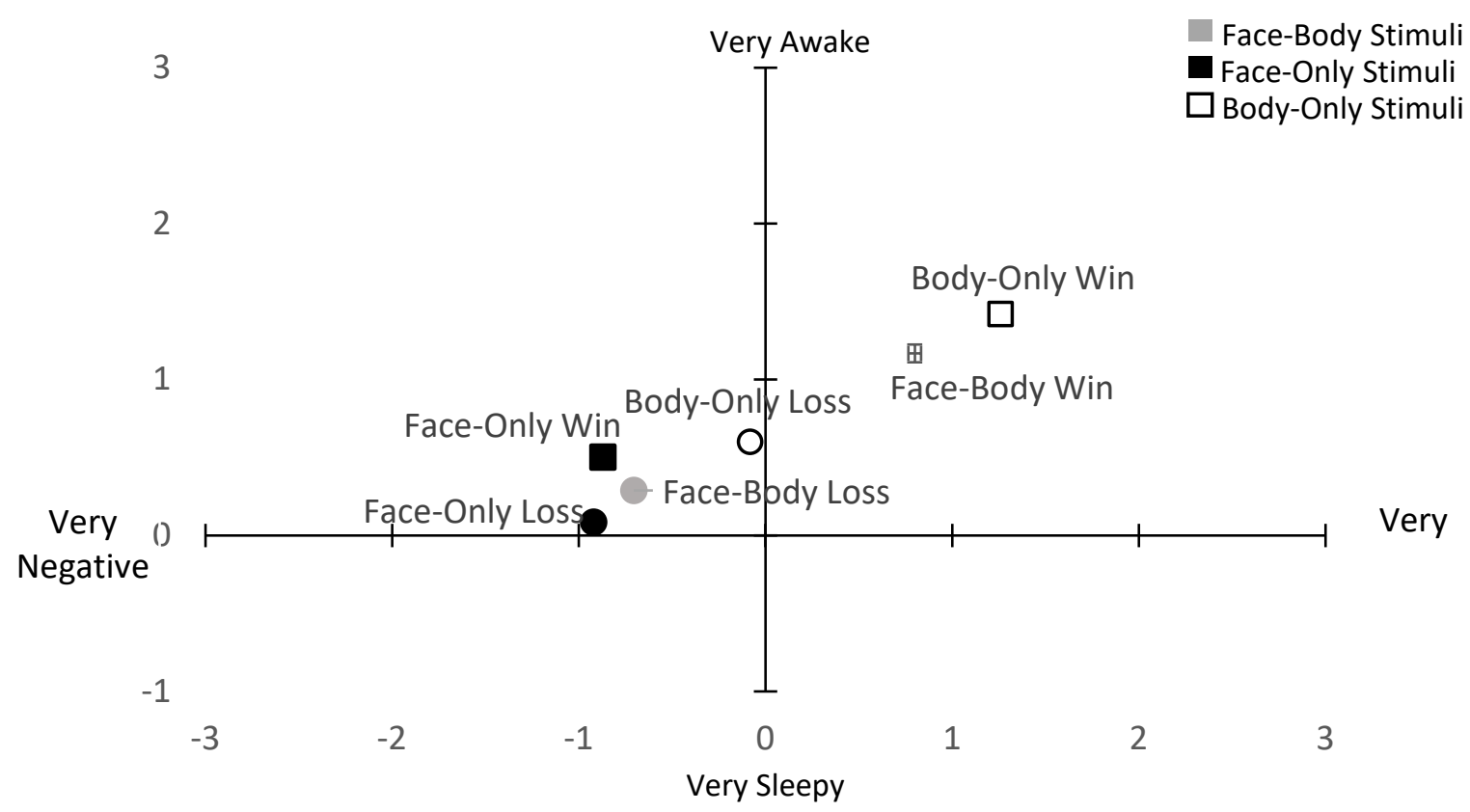

Figure 2.

Circumplex showing average valence and arousal ratings for each stimulus. Squares represent winning stimuli and circles represent losing stimuli.

\section{Valence}

A 2 (success: win, loss) x 2 (motion: dynamic, static) x 3 (stimuli type: Face-Body, Face-Only, Body-Only) repeated measures ANOVA was conducted with valence ratings as 
the dependent variable. To address violations of sphericity, Greenhouse-Geisser corrections were used where appropriate. A Bonferroni correction to the $\alpha$ level was implemented: 12 comparisons were conducted, thus we adjusted our threshold for significance to $p<.004$.

A main effect of success was identified, indicating that winning expressions $(M=$ $0.40)$ were rated more positive in valence than losing expressions $(M=-0.57), F(1,131)=$ 1509.04, $p<.001, \eta_{p}^{2}=.920$. A main effect of stimuli type, $F(2,262)=871.97, p<.001, \eta_{\mathrm{p}}{ }^{2}=$ .869 , showed that Body-Only stimuli were rated most positive $(M=0.59)$, followed by FaceBody stimuli $(M=0.05)$, and Face-Only stimuli were rated as most negative $(M=-0.90)$, $p_{\mathbf{s}}<.001$.

In support of hypothesis $1 \mathrm{~b}$, an interaction of success and stimuli type was found, $F(2$, $262)=585.63, p<.001, \eta_{\mathrm{p}}^{2}=.817$. We expected Body-only expressions to be viewed as more positive than Face-only expressions when the player had won the point, but more negative than Face-only expressions when the player lost. The results partially supported our hypotheses. As hypothesised, for winning expressions, Body-Only expressions $(\mathrm{M}=1.26)$ were perceived as more positive than Face-Only expressions $(\mathrm{M}=-.87), p<.001$. However, contrary to the hypothesis, for losing expressions, Body-Only expressions $(\mathrm{M}=-.08)$ were perceived as less negative than Face-Only $(\mathrm{M}=.92)$ expressions, $p<.001$. For both Winning and Losing expressions, Face-Body expressions were perceived as expressing moderate valence, between that of Body-Only and Face-Only expressions, $p \mathbf{s}<.001$.

The main effect of motion was not found, $p=.368$. However, in support of hypothesis $2 \mathrm{~b}$, an interaction of motion and success was identified. We expected that dynamic winning expressions would be perceived as more positive than static ones, and dynamic losing expressions would be perceived as more negative than static ones. As expected, motion intensified expression valence, $F(1,131)=100.94, p<.001, \eta_{\mathrm{p}}^{2}=.435$ (Figure X). Winning $(M=0.49)$ and losing $(M=-0.64)$ dynamic expressions displayed more positive or negative 
valence, respectively, than their corresponding winning $(M=0.30)$ and $\operatorname{losing}(M=-0.50)$ static expressions, $p \mathbf{s}<.001$.

An interaction of motion and stimuli type was found, $F(2,262)=23.471, p<.001, \eta_{p}^{2}$ $=.152$. Motion did not affect the perception of valence for Face-Body stimuli (Dynamic: $M=.08$, Static: $M=.01), p=.091$. However, motion drew perceptions of valence more toward neutral for both Face-Only (Dynamic: $M=-.79$, Static: $M=-1.00$ ), $p<.001$, and Body-Only stimuli (Dynamic: $M=.48$, Static: $M=.70$ ), $p<.001$. Therefore, the bias for perceiving FaceOnly stimuli as negatively valenced and Body-Only stimuli as positively valenced was attenuated when stimuli was dynamic compared to static.

The three-way interaction of motion, success and stimuli type was not found, $p=.864$.

\section{Accuracy}

A 2 (success: win, loss) x 2 (motion: dynamic, static) x 3 (stimuli type: Face-Body, Face-Only, Body-Only) repeated-measures ANOVA was performed. Participants' proportion of correct recognition of whether the player had won or lost was the dependent variable. To address violations of sphericity, Greenhouse-Geisser corrections were used where appropriate. A Bonferroni correction was implemented: 17 comparisons were conducted, and our adjusted threshold for significance was $p<.003$.

A main effect of stimuli type was identified, $F(2,262)=292.73, p<.001, \eta_{\mathrm{p}}{ }^{2}=.69$ (Figure 3a.). In support of hypothesis 1a, participants were more accurate in determining whether a player had won or lost when they viewed Body-Only stimuli than Face-Only stimuli, $p<.001$. Additionally, participants were more accurate for Face-Body stimuli $(M=$ $.72)$ than either Face-Only $(M=.54)$ or Body-Only $(M=.68)$ stimuli, $p \mathbf{s}<.001$. This complements our finding with valence ratings in which Face-Body stimuli were best recognized, followed by Body-Only stimuli, and lastly Face-Only stimuli. 
In support of hypothesis 2a, a main effect of motion was also identified. It was found that more accurate judgements were made for dynamic stimuli $(M=.66)$ than static stimuli $(M=.63), F(1,131)=19.24, p<.001, \eta_{p}^{2}=.13$ (Figure 3b). Again, this supports our previous results found with valence judgements showing that dynamic expressions were viewed as more positive for wins and more negative for losses than static expressions.

a) 1

0.9

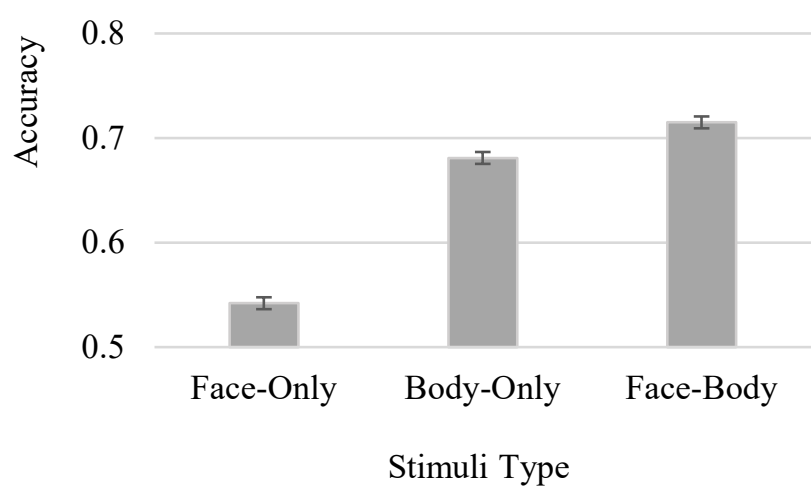

b)

$$
0.9
$$

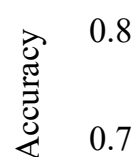

0.6

0.5

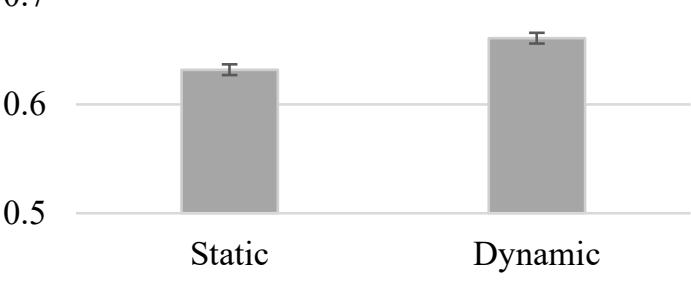

Motion of Stimuli

Figure 3. Mean Accuracy Judgements for a) Face-Only, Body-Only, and Face-Body forms, and for b) Static and Dynamic expressions. Error bars represent Standard Error.

A main effect of success was found showing participants were more accurate in determining that losing players had lost $(M=.73)$ than that winning players had won $(M=$ .56), suggesting a bias to see expressions as losses, $F(1,131)=131.76, p<.001, \eta_{\mathrm{p}}{ }^{2}=.501$. The two-way interaction between success and stimuli type, $F(2,262)=440.22, p<.001, \eta_{\mathrm{p}}{ }^{2}=$ .771 , was followed up with post-hoc analyses comparing winning and losing expressions at each level of stimulus type. For Face-Body and Face-Only stimuli, participants more accurately recognized losing expressions than winning ones (Mace-Body: .79 vs. .65; M Body-only: .79 vs. .29), $p \mathrm{~s}<.001$. Conversely, for Body-Only stimuli participants more accurately recognised wins $(M=.75)$ than losses $(M=.62), p<.001$. This finding aligns with our earlier 
result showing that Body-Only stimuli were perceived as more positively valenced than FaceOnly stimuli.

The interaction of motion and success was not found, $F(1,131)=.372, p=.543, \eta_{p}^{2}=$ .003. The two-way interaction of motion and stimuli type, and the three-way interaction of motion, success and stimuli type were both significant but demonstrated small effects, $p \mathrm{~s}=.015, .008, \eta_{p}{ }^{2} \mathrm{~s}=.032, .036$, respectively. As these effects were both small and exploratory, we choose not to detail follow up analyses here. Further detail regarding these effects may be found in the Supplementary Materials.

\section{Arousal}

A 2 (success: win, loss) x 2 (motion: dynamic, static) x 3 (stimuli type: Face-Body, Face-Only, Body-Only) repeated measures ANOVA was conducted with arousal Likert ratings as the Dependent Variable. In cases where sphericity was violated GreenhouseGeisser corrections were used. Again, to manage the potential elevation of the family-wise error rate due to multiple post-hoc comparisons, a Bonferroni correction to the $\alpha$ level was implemented. As 12 comparisons were conducted, $p<.050$ was adjusted to $p<.004$ as a threshold for significance. No directional hypotheses were pre-registered for arousal and all analyses are exploratory.

A main effect of stimuli type, $F(2,262)=136.28, p<.001, \eta_{p}^{2}=.514$, showed that Body-Only expressions $(M=1.02)$ were rated as higher in arousal than Face-Body expressions $(M=.73), p<.001$, and Face-Only expressions rated as lowest in arousal $(M=$ $.29), p \mathrm{~s}<.001$.

A main effect of success was identified such that winning expressions $(M=1.04)$ were perceived as higher in arousal than losing expressions $(M=.32), F(1,131)=.763, p=.384, \eta_{p}^{2}$ $=.006$. 
No main effect of motion was identified, $F(1,131)=.763, p=.384, \eta_{p}^{2}=.006$.

However, a two-way interaction of motion and success was found, $F(1,131)=31.77, p<.001$, $\eta_{p}{ }^{2}=.195$. Motion increased perceptions of arousal for winning expressions (Dynamic: $M=1.11$, Static: $M=.97$ ) but not for losing expressions (Dynamic: $M=.28$, Static: $M=.36$ ), $p<.001, p=.070$, respectively.

An interaction of motion and stimuli type was also found, $F(2,262)=17.26, p<.001$, $\eta_{\mathrm{p}}{ }^{2}=.116$. Dynamic Face-Only expressions $(M=.40)$ were rated as higher in arousal than static Face-Only expressions $(M=.18), p<.001$. However, dynamic Body-Only expressions $(M=.94)$ were rated as lower in arousal than static Body-Only expressions $(M=1.09)$, $p<.001$. Dynamic and static Face-Body expressions did not differ $(M=.74 ; M=.72$, respectively), $p=.642$.

An interaction of success and stimuli type was found, $F(2,262)=53.92, p<.001, \bigcap_{p}{ }^{2}$ $=.292$. For all stimuli types, winning expressions (Face-Body: $M=1.17$, Face-Only: $M=.50$, Body-Only: $M=1.44$ ) were perceived as higher in arousal than losing expressions (FaceBody: $M=.29$, Face-Only: $M=.08$, Body-Only: $M=.58), p \mathbf{s}<.001$. However, the difference between the arousal ratings for winning and losing Face-Body and Body-Only expressions (Mean differences $=.88, .87$, respectively) was approximately twice as large as that for FaceOnly expressions (Mean difference $=.42$ ).

\section{Discussion}

The aim of this study was to explore how naturalistic, dynamic, whole person emotion expressions are perceived, expanding on the research of Aviezer et al. (2012a). Although we selected a broader range of expressions than Aviezer et al. (2012a) did, incorporating dynamic expressions and selecting expressions based on context rather than intensity, we found their results largely replicated. This suggests that Aviezer et al.'s pattern of findings are robust and apply to a range of expressive behaviour. Our inclusion of dynamic expressions 
allowed us to expand upon their work, however, and make several comparisons regarding the effect of motion on emotion perception for naturalistic emotion expressions.

\section{The Effect of Cue Type}

As hypothesised, perceptions of bodily expressions were more accurate than facial expressions. This was supported by converging evidence across our measures of accuracy and valence. Body-only expressions were more accurately identified as wins and losses than Face-only expressions, and valence ratings were more divergent for winning and losing Body-only expressions than Face-only ones. Of particular interest, participant's perception of valence for winning and losing faces did not differ, suggesting faces were particularly uninformative, and may not convey interpretable information regarding valence differences. Our findings add to a growing body of research showing poor emotion recognition of naturalistic facial expressions, and an increase in recognition when postural expressions are present(Abramson et al., 2017; J Duran et al., 2017; Fernández-Dols \& Crivelli, 2013; Reisenzein et al., 2013; Wenzler et al., 2016). These findings, along with our own, highlight the need for further research examining the naturalistic expression of emotion.

As with prior research, we found that when more cues were available to participants emotion recognition increased. In this case, as in previous research (e.g., Aviezer et al, 2012a), when participants judged the face and body together, they were more likely to accurately perceive whether the player had won or lost and the valence of the expression.

Exploratory analyses found that expressions from faces were perceived as more negatively valenced and bodies were perceived as more positively valenced. This was supported by evidence from the accuracy judgements, where faces were more often believed to be losing expressions, and bodies were more often believed to be winning expressions. The bias to perceive faces as more negatively valenced and bodies as more positively valenced replicates the work of Aviezer et al. (2012a) but to our knowledge has not been found in other 
studies examining naturalistic expressions. Thus, it is possible that this finding is contextually specific to professional sporting matches or may be linked to the physical exertion experienced by players. Future research might consider examining naturalistic expressions in a wider range of contexts.

\section{The Effect of Motion}

As previous research has found motion improves emotion perception (Ambadar et al., 2005; Atkinson et al., 2004; Krumhuber et al., 2013; Nelson \& Mondloch, 2017), we expected to observe a similar pattern in our data, with emotion perception being improved when stimuli were presented dynamically rather than statically. This hypothesis was supported, with dynamic winning expressions more often identified as wins and perceived as more positively valenced than static expressions, and dynamic losing expressions more often identified as losses and perceived more negatively valenced than static expressions. Additionally, motion moderated participants' bias to see faces as negative and bodies as positive: when stimuli were presented dynamically, participants were more likely to see faceand body-only expressions as more similarly valenced. Specifically, dynamic winning faces were judged as more positively valenced and dynamic losing bodies judged as more negatively valenced than their static counterparts.

These findings align with previous research indicating that motion can improve emotion perception (Ambadar et al., 2005; Atkinson et al., 2004; Krumhuber et al., 2013; Nelson \& Mondloch, 2017). However, to our knowledge, our results are the first evidence that this pattern persists with naturalistic expressions.

\section{Arousal}

We did not have specific, directional hypotheses related to arousal ratings, due to limited previous research examining arousal ratings of naturalistic expressions. However, some previous research has suggested that dynamic expressions may be perceived as being 
higher in arousal than static ones (Barker et al., 2020; Krumhuber et al., 2013). However, this was not found in our study. An interaction was identified between motion and success, indicating that winning dynamic expressions were viewed as higher in arousal compared to static ones, but no difference was found between losing dynamic and static expressions. Given the exploratory nature of our analysis, and the fact that these effects were relatively small, our results do not support the assertion that motion increases perceived arousal. Additional evidence from other naturalistic expressions is needed to determine whether the absence of this effect is due to the specific context of tennis matches or due to differences in naturalistic and posed expressions.

\section{Strengths, Limitations and Futures Research}

Our study used a single, emotionally intense situation to gather examples of naturalistic emotional expressions. This, of course, is a narrow and extreme emotional context, but allowed us to conduct a rigorous analysis of how movement and expression cues influence participant's affective judgments of genuine expressions. Our findings diverge from those generally found using posed expressions in important ways and support previous arguments that the narrow range of information displayed in posed facial expressions fails to capture the diversity of behaviours demonstrated in real-world emotional expressions (Fernández-Dols \& Crivelli, 2013). Future research should continue to seek varied, ecologically valid examples of naturalistic emotion expression. Only by documenting and including the diversity of genuine emotion expressive behaviour from naturalistic settings can theory and literature provide a more complete understanding of typical emotion expressive behaviour.

\section{Conclusions}

Natural emotion expression is dynamic and expressed through a variety of cues, including the face and the body. This research demonstrates that, for naturalistic emotion 
expression, presenting expressions as dynamic and with the whole person visible increases emotion recognition. The use of static, posed, isolated facial expressions limits valuable information people rely on to judge other's emotional expressions. We argue that future research must incorporate more ecologically valid expressions to ensure the findings of laboratory-based emotion research generalise beyond the laboratory. 


\section{Compliance with Ethical Standards:}

Ethical approval: All procedures performed in studies involving human participants were in accordance with the ethical standards of the institutional and/or national research committee and with the 1964 Helsinki declaration and its later amendments or comparable ethical standards.

Informed consent: Informed consent was obtained from all individual participants included in the study. 


\section{References}

Abramson, L., Marom, I., Petranker, R., \& Aviezer, H. (2017). Is fear in your head? A comparison of instructed and real-life expressions of emotion in the face and body. Emotion, 17(3), 557-565. https://doi.org/10.1037/emo0000252

Ambadar, Z., Schooler, J. W., \& Cohn, J. F. (2005). Deciphering the enigmatic face: The importance of facial dynamics in interpreting subtle facial expressions. Psychological Science, 16(5), 403-410. https://doi.org/10.1111/j.0956-7976.2005.01548.x

Atkinson, A. P., Dittrich, W. H., Gemmell, A. J., \& Young, A. W. (2004). Emotion perception from dynamic and static body expressions in point-light and full-light displays. Perception, 33(6), 717-746. https://doi.org/10.1068/p5096

Aviezer, H., Dudarev, V., Bentin, S., \& Hassin, R. R. (2011). The automaticity of emotional face-context integration. Emotion, 11(6), 1406-1414.

https://doi.org/10.1037/a0023578.The

Aviezer, H., Ensenberg, N., \& Hassin., R. R. (2017). The inherently contextualized nature of facial emotion perception. Current Opinion in Psychology, 17, 47-54.

https://doi.org/10.1016/j.copsyc.2017.06.006

Aviezer, H., Ensenberg, N., \& Hassin, R. R. (2017). The inherently contextualised nature of facial emotion perception. Current Opinion in Psychology, 17, 47-54.

Aviezer, H., Trope, Y., \& Todorov, A. (2012a). Body Cues, Not Facial Expressions, Discriminate Between Intense Positive and Negative Emotions. Science, 338(November 2012), 1225-1230. https://www.semanticscholar.org/paper/Aviezer-Positive-andNegative-Emotions-Body-Cues-\%2C-Rana-

Kakuda/6101d0fd6f00679d56b60c12d4f97377ebc29cf6

Aviezer, H., Trope, Y., \& Todorov, A. (2012b). Holistic person processing: Faces with bodies tell the whole story. Journal of Personality and Social Psychology, 103(1), 20- 
37. https://doi.org/10.1037/a0027411

Barker, M. S., Bidstrup, E. M., Robinson, G. A., \& Nelson, N. L. (2020). “Grumpy” or “furious”? arousal of emotion labels influences judgments of facial expressions. PloS One, 15(7), e0235390. https://doi.org/10.1371/journal.pone.0235390

Barrett, L. F., Adolphs, R., Marsella, S., Martinez, A. M., \& Pollak, S. D. (2019). Emotional Expressions Reconsidered: Challenges to Inferring Emotion From Human Facial Movements. Psychological Science in the Public Interest, 20(1), 1-68. https://doi.org/10.1177/1529100619832930

Bould, E., \& Morris, N. (2008). Role of motion signals in recognizing subtle facial expressions of emotion. British Journal of Psychology, 99, 167-189. https://doi.org/10.1348/000712607X206702

Cowen, A. S., \& Keltner, D. (2019). What the Face Displays: Mapping 28 Emotions Conveyed by Naturalistic Expression. American Psychologist. https://doi.org/10.1037/amp0000488

De Gelder, B. (2009). Why bodies? Twelve reasons for including bodily expressions in affective neuroscience. Philosophical Transactions of the Royal Society B: Biological Sciences, 364(1535), 3475-3484. https://doi.org/10.1098/rstb.2009.0190

Douglas-Cowie, E., Cox, C., Martin, J. C., Devillers, L., Cowie, R., Sneddon, I., McRorie, M., Pelachaud, C., Peters, C., Lowry, O., Batliner, A., \& Hönig, F. (2011). The HUMAINE database. In Emotion Oriented Systems (Issue 9783642151835, pp. 243284). https://doi.org/10.1007/978-3-642-15184-2_14

Dukes, D., Clément, F., Audrin, C., \& Mortillaro, M. (2017). Looking beyond the static face in emotion recognition: The informative case of interest. Visual Cognition, 25(4-6), 575-588. https://doi.org/10.1080/13506285.2017.1341441

Duran, J, Reisenzein, R., \& Fernández-Dols, J. M. (2017). Coherence Between Emotions and 
Facial Expressions. In The Science of Facial Expressions.

https://doi.org/10.1093/acprof:oso/9780190613501.003.0007

Duran, Juan., \& Fernández-Dols, J. M. (2018). Do Emotions Result in their Predicted Facial Expressions? A Meta-Analysis of Studies on the Link between Expression and Emotion. PsyArXiv Preprints, 118-121.

Faul, F., Erdfelder, E., Lang, A.-G., \& Buchner, A. (2007). G*Power 3: A flexible statistical power analysis program for the social, behavioral, and biomedical sciences. Behaviour Research Methods, 39(2), 175-191. https://doi.org/10.3758/BF03193146

Fernández-Dols, J. M., \& Crivelli, C. (2013). Emotion and expression: Naturalistic studies. Emotion Review, 5(1), 24-29. https://doi.org/10.1177/1754073912457229

Fernández-Dols, J. M., Sanchez, F., Carrera, P., \& Angeles, R.-B.-M. (1997). Are Spontaneous Expressions and Emotions Linked? An Experimental Test of Coherence. Journal of Nonverbal Behavior, 21(3), 163-177. https://doi.org/10.1007/BF01335828

Hess, U., \& Kleck, R. E. (1990). Differentiating emotion elicited and deliberate emotional facial expressions. European Journal of Social Psychology, 20(5), 369-385. https://doi.org/10.1002/ejsp.2420200502

Kraut, R. E., \& Johnston, R. E. (1979). Social and emotional messages of smiling: An ethological approach. Journal of Personality and Social Psychology, 37(9), 1539-1553. https://doi.org/10.1037/0022-3514.37.9.1539

Krumhuber, E. G., Kappas, A., \& Manstead, A. S. R. (2013). Effects of dynamic aspects of facial expressions: A review. Emotion Review, 5(1), 41-46. https://doi.org/10.1177/1754073912451349

Krumhuber, E. G., Skora, L., Küster, D., \& Fou, L. (2017). A Review of Dynamic Datasets for Facial Expression Research. Emotion Review, 9(3), 280-292. https://doi.org/10.1177/1754073916670022 
Lecker, M., Shoval, R., Aviezer, H., \& Eitam, B. (2017). Temporal integration of bodies and faces: united we stand, divided we fall? Visual Cognition, 1-15. https://doi.org/10.1080/13506285.2017.1310164

Martinez, L., Falvello, V. B., Aviezer, H., \& Todorov, A. (2016). Contributions of facial expressions and body language to the rapid perception of dynamic emotions. Cognition and Emotion, 30(5), 939-952. https://doi.org/10.1080/02699931.2015.1035229

Mondloch, C. J., Nelson, N. L., \& Horner, M. (2013). Asymmetries of influence: Differential effects of body postures on perceptions of emotional facial expressions. PLoS ONE, 8(9), 1-16. https://doi.org/10.1371/journal.pone.0073605

Namba, S., Kabir, R. S., Miyatani, M., Nakao, T., \& Douglas, K. (2018). Dynamic Displays Enhance the Ability to Discriminate Genuine and Posed Facial Expressions of Emotion. 9(May), 1-12. https://doi.org/10.3389/fpsyg.2018.00672

Namba, S., Makihara, S., Kabir, R. S., Miyatani, M., \& Nakao, T. (2017). Spontaneous Facial Expressions Are Different from Posed Facial Expressions: Morphological Properties and Dynamic Sequences. Current Psychology, 36(3), 593-605.

https://doi.org/10.1007/s12144-016-9448-9

Namba, S., Matsui, H., \& Zloteanu, M. (2021). Distinct temporal features of genuine and deliberate facial expressions of surprise. Scientific Reports, 1-10. https://doi.org/10.1038/s41598-021-83077-4

Nelson, N. L., \& Mondloch, C. J. (2017). Adults' and children's perception of facial expressions is influenced by body postures even for dynamic stimuli. Visual Cognition, 1-12. https://doi.org/10.1080/13506285.2017.1301615

Reisenzein, R., Bördgen, S., Holtbernd, T., \& Matz, D. (2006). Evidence for strong dissociation between emotion and facial displays: The case of surprise. Journal of Personality and Social Psychology, 91(2), 295-315. https://doi.org/10.1037/0022- 
3514.91.2.295

Reisenzein, R., Studtmann, M., \& Horstmann, G. (2013). Coherence between emotion and facial expression: Evidence from laboratory experiments. Emotion Review, 5(1), 16-23. https://doi.org/10.1177/1754073912457228

Ruiz-Belda, M. A., Fernández-Dols, J. M., Carrera, P., \& Barchard, K. (2003). Spontaneous facial expressions of happy bowlers and soccer fans. Cognition and Emotion, 17(2), 315-326. https://doi.org/10.1080/02699930302288

Sauter, D. A., \& Fischer, A. H. (2018). Can perceivers recognise emotions from spontaneous expressions? Cognition and Emotion, 32(3), 504-515. https://doi.org/10.1080/02699931.2017.1320978

Schützwohl, A., \& Reisenzein, R. (2012). Facial expressions in response to a highly surprising event exceeding the field of vision: A test of Darwin's theory of surprise. Evolution and Human Behavior, 33(6), 657-664. https://doi.org/10.1016/j.evolhumbehav.2012.04.003

Wenzler, S., Levine, S., van Dick, R., Oertel-Knöchel, V., \& Aviezer, H. (2016). Beyond pleasure and pain: Facial expression ambiguity in adults and children during intense situations. Emotion, 16(6), 807-814. https://doi.org/10.1037/emo0000185

Zloteanu, M., Krumhuber, E. G., Richardson, D. C., \& Livingstone, S. R. (2018). Detecting Genuine and Deliberate Displays of Surprise in Static and Dynamic Faces. 9(July), 1-9. https://doi.org/10.3389/fpsyg.2018.01184 